\title{
Study of the degradation of olive oil (olea europaea) by optical spectroscopy
}

\author{
Juliane Mendonça Pereira ${ }^{1}$, Petrus Alcântara Junior ${ }^{2}$
}

\begin{abstract}
${ }^{1}$ Programa de Formação Interdisciplinar de Meio Ambiente do Núcleo de Meio Ambiente (NUMA). Universidade Federal do Pará (UFPA). Campus do Guamá, 66075-110, Belém, PA, Brasil Fone: (55-91)32017421.

${ }^{2}$ Programa de Pós-Graduação em Física e Programa de Pós-Graduação em Ciências e Meio Ambiente (PPGCMA). Instituto de Ciências Exatas e Naturais da Universidade Federal do Pará (ICEN-UFPA). Campus do Guamá, Belém, PA, Brasil, 66075-110. Fone: (5591)32017421.
\end{abstract}

Email: ju_spm@yahoo.com.br, petrus@ufpa.br

Received: January $17^{\text {th }}, 2017$

Accepted: February $10^{\text {th }}, 2017$

Published: March 30th 2017

Copyright (C2016 by authors and Institute of Technology Galileo of Amazon (ITEGAM) This work is licensed under the Creative Commons Attribution International License (CC BY 4.0).

http://creativecommons.org/licenses/by/4.0/ (c) (1) (3) Open Actes:

\begin{abstract}
UV-VIS optical spectroscopy was applied to samples of olive oil used in frying potatoes at $180^{\circ} \mathrm{C}$ at regular intervals of fifteen minutes for seven consecutive days in order to analyze changes suffered by minority pigments that has much appeal to the quality properties of olive oil. The degradation of these pigments can contribute greatly to the oxidation of the oil, so the acidity and peroxide indices of each sample were measured simultaneously. The spectra show a shift towards blue and decreasing in absorption intensities, as frying time increases, in the regions of absorption of $\beta$-carotene and chlorophyll A. Comparison of results suggests that changes occurring with pigments contribute to the increase of the acidity index. This indicates that spectroscopy can be used as a simple, efficient and non-invasive technique for monitoring the quality of olive oil.
\end{abstract}

Key-Words: Olive oil. Degradation. Optical Spectroscopy. B-carotene. Chlorophyll.

\begin{abstract}
RESUMO
Espectroscopia óptica UV-VIS foi aplicada a amostras de azeite de oliva utilizado em fritura de batatas a $180^{\circ} \mathrm{C}$ por intervalos regulares de quinze minutos, durante sete dias consecutivos a fim de analisar alterações sofridas por pigmentos minoritários que tem muito apelo às propriedades de qualidade do azeite de oliva. A degradação desses pigmentos pode contribuir fortemente para a oxidação do azeite, portanto foram medidos simultaneamente os índices de acidez e de peróxido de cada amostra. Os espectros mostram um deslocamento para o azul e diminuição nas intensidades de absorção, à medida que o tempo de fritura aumenta, nas regiões de absorção de $\beta$-caroteno e de clorofila A. Comparação dos resultados sugere que as alterações ocorridas com os pigmentos contribuem para o aumento do índice de acidez. Isto indica que espectroscopia pode ser utilizada como uma técnica simples, eficiente e não invasiva no monitoramento da qualidade de azeite de oliva.
\end{abstract}

Palavras-Chave: Azeite de oliva. Degradação. Espectroscopia Óptica. B-caroteno. Clorofila.

\section{INTRODUÇÃO}

Os óleos vegetais ocupam um lugar privilegiado na indústria, e sua utilização como alimento é necessária porque apresentam em sua constituição componentes moleculares importantes no metabolismo humano, como ácidos graxos essenciais que não são produzidos pelo organismo. Estão envolvidos no abastecimento e no armazenamento de energia; relacionados à síntese de hormônios; componentes da bile e da membrana celular. Um dos óleos vegetais mais consumidos no mundo é o azeite de oliva (Olea europaea) por causa de suas propriedades relevantes à saúde humana, sendo apontado como 
responsável por prevenção de doenças cardiovasculares e obesidade, entre outras [1]. O azeite de oliva é composto, majoritariamente, por ácidos graxos saturados, como o ácido palmítico (C16:0), o ácido esteárico (C18:0) e outros em menores quantidades, e insaturados como o ácido oleico (C18:1) que está presente em maior proporção, com teores entre 55-83\%, e ácidos graxos essenciais como o ácido linoleico (C18:2) e ácido linolênico (C18:3). Também estão presentes em sua composição esqualenos e $\beta$-caroteno, o primeiro é parcialmente responsável pelos efeitos benéficos à saúde humana, particularmente na prevenção de alguns tipos de cânceres [2], enquanto o último é responsável pela coloração amarelo-esverdeada do azeite [3]. Encontram-se ainda em sua composição química $\alpha$-tocoferol e, preponderantemente clorofila A [4].

Os pigmentos presentes no azeite de oliva ( $\beta$-caroteno e clorofila), além de atribuírem a cor característica ao produto são fatores relevantes no que se refere aos processos oxidativos, a clorofila atuando como fotossensibilizador acelerando o processo de oxidação e o $\beta$-caroteno na qualidade de inibidor natural, sendo assim um forte antioxidante[5]. Quanto a clorofila, em processos que envolvem luz e calor, ocorre a produção do oxigênio singleto que, reagindo com ácidos graxos, produzem hidroperóxidos e a partir destes há um desencadeamento da auto-oxidação, com formação de radicais livres. No mecanismo de proteção dos constituintes químicos do azeite, o $\beta$-caroteno tem o papel relevante de impedir a evolução das reações oxidativas, notadamente em presença de luz $[5,6]$.

Em geral se dá muita importância ao consumo do azeite in natura, mas o mesmo também pode ser usado em fritura de alimentos. Damy et al [7] apontam que a fritura de alimentos é uma operação importante por ser um processo de preparação rápida e por conferir aos produtos fritos características únicas de odor e paladar. Nesse processo, o óleo interage com o ar, água e componentes dos alimentos que estão sendo fritos gerando compostos desagradáveis e degradações em óleos utilizados por longos períodos. Nesse sentido, experimentos foram conduzidos em laboratório utilizando fritura descontínua de batatas para avaliar o comportamento físico-químico do azeite de oliva, à temperatura de $180^{\circ} \mathrm{C}$.

A reutilização frequente do óleo na fritura pode levar a níveis mais elevados de oxidação, sendo possível detectar inibidores enzimáticos, destruidores de vitaminas; produtores de oxidação lipídica, irritantes gastrointestinais e agentes mutagênicos ou cancerígenos, além de oxidação do bom colesterol, aumentando o risco do desenvolvimento de doenças cardiovasculares [7].

O acompanhamento da degradação de óleos em geral tem motivado estudos e controles de qualidade, que em geral são feitos por métodos químicos de titulação, como os índices de acidez e de peróxido; índice de iodo e saponificação, e/ou por medidas de propriedades físicas, como medidas de viscosidade e índice de refração, entre outros. Apesar de serem os métodos mais usuais, sua confiabilidade e precisão dependem de repetições, tornando os procedimentos demorados e de alto custo [8].

Espectroscopia UV-VIS tem sido utilizada na investigação da composição química de óleos vegetais e, em particular, de suas alterações devido a efeitos externos como temperatura e armazenamento. No primeiro caso, Albuquerque et al [9] reportaram de forma qualitativa da composição química do óleo de buriti identificando seus principais constituintes moleculares nas regiões do UV-VIS-NIR. No caso particular do azeite de oliva, Domenici et al [4] fizeram um estudo usando espectroscopia UV-VIS dos pigmentos mais frequentes na composição do azeite: clorofilas e carotenoides, apontando para a degradação de alguns desses componentes por ação da temperatura por longo tempo de exposição. Muitos pesquisadores têm visto nesse método a resposta para o problema do monitoramento rápido e preciso da degradação nutricional de azeites de oliva.

Motivados pelos problemas que a ingestão de azeites degradados pode causar à saúde humana, neste trabalho descrevemos o uso da espectroscopia óptica para azeite de oliva após ser utilizado em processos de fritura, analisando as assinaturas espectrais de $\beta$-caroteno e clorofila A como indicadores de perda de qualidade em virtude da relação direta de tais componentes para a ação oxidativa do azeite; estas análises são complementadas por métodos químicos tradicionais de análise quantitativa da oxidação de óleos vegetais.

\section{II.MATERIAIS E MÉTODOS}

Foi usado azeite de oliva comercial do tipo extra virgem marca Gallo adquirido no mercado local. Segundo indicação na embalagem, o óleo foi fabricado em 09/2013, com validade até 03/2015, pertencendo ao lote 3259 H8388 14:43. Segundo informações do fabricante, contidas na embalagem, a acidez máxima é maior ou igual a 1,00\% e o índice de peróxido, maior ou igual a 15,00 meq/kg, valores normais segundo regulamentação da Agência Nacional de Vigilância Sanitária (ANVISA), contida na Resolução n $n^{0}$ 482, de 23/09/1999.

A temperatura média utilizada na fritura de batatas foi $180^{\circ} \mathrm{C}$ [7].

$\mathrm{O}$ azeite foi usado durante sete dias consecutivos a intervalos de tempo 15 minutos por dia, tempo suficiente para atingir a temperatura desejada e obter o produto final. Os processos foram realizados em cozinha comum, afim de se retratar uma atividade cotidiana, com a presença de fatores como luz, calor, ar, etc. Após cada fritura observa-se uma diminuição no volume de azeite, devido a competição entre a evaporação e a absorção pela batata, além da quantidade de $50 \mathrm{ml}$ retirada para efetuar as análises. Não havendo reposição do azeite, estabeleceu-se uma relação direta entre a massa de batata a ser frita e a quantidade de azeite restante a cada dia da fritura, como mostra a tabela 1:

Tabela 1: Fritura - Batata x Azeite.

\begin{tabular}{c|r|c}
\hline Dia de fritura & Batata $(\mathrm{g})$ & Azeite de oliva $(\mathrm{ml})$ \\
\hline Primeiro & 250 & 900 \\
\hline Segundo & 217 & 780 \\
\hline Terceiro & 183 & 660 \\
\hline Quarto & 150 & 540 \\
\hline Quinto & 100 & 360 \\
\hline Sexto & 75 & 270 \\
\hline Sétimo & 58 & 210
\end{tabular}

Fonte: Os Autores, 2016.

Para realização do processo, utilizou-se de: panelas de alumínio com $30 \mathrm{~cm}$ de diâmetro e $15 \mathrm{~cm}$ de altura para as frituras; termômetro de mercúrio com escala de 0 a $300^{\circ} \mathrm{C}$, cronômetro para monitoramento do tempo de fritura estabelecido (15 min); balança comercial para verificação da massa de batata; colher de alumínio para manuseio do alimento; béquer de $50 \mathrm{ml}$ para armazenamento inicial das amostras; fogão doméstico; geladeira; régua para medição da altura da coluna de óleo; batata e tubo de centrifugação $50 \mathrm{ml}$ para o armazenamento final, após o resfriamento.

Armazenou-se as amostras coletadas em local fechado fora do alcance da luz, a uma temperatura ambiente de 
aproximadamente $28^{\circ} \mathrm{C}$. Após a preparação de todas as amostras, as mesmas foram submetidas a análises quanto ao índice de acidez, índice de peroxido e por espectroscopia óptica.

\section{1 ÍNDICE DE ACIDEZ}

O índice de acidez foi estabelecido por meio de método usual e normas rigorosamente estabelecidas para o alcance preciso de seus resultados, em $\mathrm{mg} \mathrm{NaOH} / \mathrm{g}$ [10].

Para as análises usou-se uma balança analítica marca EQUISUL, modelo SHIMADZU; erlenmeyer $125 \mathrm{ml}$; proveta 25 $\mathrm{ml}$; bureta de $25 \mathrm{ml}$, bastão de vidro, balão volumétrico, espátula e placa de Petri, estufa.

Reagentes: Álcool etílico (NUCLEAR); fenolftaleína (NUCLEAR); hidróxido de sódio e biftalato de potássio (NUCLEAR).

\section{2 ÍNDICE DE PERÓXIDO}

Assim como o índice de acidez, a determinação do índice de peróxido, em meq $/ \mathrm{kg}$, também foi realizado segundo normas pré-estabelecidas [10]. Para realização das análises usou-se: Balança analítica; erlenmeyer de $125 \mathrm{ml}$ ou $250 \mathrm{ml}$ com tampa esmerilhada; proveta de $25 \mathrm{ml}$; pipeta graduada de $1 \mathrm{ml}$; bureta de $25 \mathrm{ml}$; chapa térmica vibratória.

Reagentes: Ácido acético (NOX); dicromato de potássio (NUCLEAR); solução de tiossulfato de sódio $0,1 \mathrm{~N}$ (laboratório VETEC); solução de amido solúvel; iodeto de potássio (DINÂMICA); solução de ácido acético-clorofórmio (3:2), clorofórmio (NOX) e ácido acético (NOX).

\section{II.3 ESPECTROSCOPIA DE ABSORÇÃO}

Os espectros de absorção foram feitos com espectrofotômetro JASCO V-670 com varredura de 190 a 2700nm e precisão mínima de $0,1 \mathrm{~nm}$, controlado automaticamente por computador usando o programa SpectraManager que também foi responsável pela aquisição dos espectros. Para realização dos espectros usou-se cubetas KARTELL S.P.A. com capacidade de $4,5 \mathrm{ml}$.

Espectroscopia UV-VIS já foi empregada em trabalhos anteriores como o objetivo de identificar constituintes moleculares de óleos vegetais de diferentes fontes.

Realizaram-se analises por espectroscopia de absorção no comprimento de 300-1600nm, entretanto os componentes moleculares que apresentaram modificações mais significativas ao longo do processo de fritura se encontravam na região entre 375700nm. Assim está região foi selecionada para o estudo em questão.

\section{RESULTADOS E DISCUSSÕES}

\section{III.1. ÍNDICE DE ACIDEZ}

A tabela 2 mostra os valores dos índices de acidez medidos durante sete dias de fritura. A indexação das amostras em numerações de 0 a 7 correspondem aos dias em que foram realizadas as frituras, sendo que 0 indica o azeite de oliva antes de qualquer aquecimento. A medida da amostra zero representa tem um valor de $0,90 \mathrm{mg} \mathrm{NaOH} / \mathrm{g}$ ), apresentando-se dentro do padrão recomendado pela ANVISA [11], que é de até $1,0 \mathrm{mg} \mathrm{NaOH} / \mathrm{g}$.

Tabela 2: Amostra - Tempo de fritura x índice de acidez.

\begin{tabular}{c|c|c}
\hline Amostra & $\begin{array}{l}\text { Tempo de } \\
\text { fritura (min) }\end{array}$ & $\begin{array}{c}\text { Índice de acidez } \\
(\mathrm{mg} \mathrm{NaOh} / \mathrm{g})\end{array}$ \\
\hline 0 & 0 & 0,90 \\
\hline 1 & 15 & 1,50 \\
\hline 2 & 30 & 1,80 \\
\hline 3 & 45 & 1,80 \\
\hline 4 & 60 & 1,80 \\
\hline 5 & 75 & 1,85 \\
\hline 6 & 90 & 2,15 \\
\hline 7 & 105 & 2,20 \\
\hline
\end{tabular}

Fonte: Os Autores, 2016.

Observa-se que o índice de acidez aumentou ao longo dos dias, o que sugere a formação de radicais livres e o aumento do número destes ao longo do tempo. Esse aumento já era esperado, uma vez que as exposições do azeite a temperaturas elevadas, assim como seu contato com o ar e a água contidos nos alimentos, contribuem para a quebra dos ácidos graxos e consequente formação de radicais livres. Resultados similares foram reportados em outros trabalhos $[12,13]$.

\section{III.2. ÍNDICE DE PERÓXIDO}

A tabela 3 traz os valores dos índices de peróxido do azeite estudado em intervalos de tempo de 15 minutos (fritura), durante sete dias. $\mathrm{O}$ tempo zero corresponde a amostra antes do processo.

Tabela 3: Índices de peróxido para o azeite de oliva na fritura.

\begin{tabular}{c|c|c}
\hline Amostra & $\begin{array}{c}\text { Tempo de } \\
\text { fritura (min) }\end{array}$ & $\begin{array}{c}\text { Índice de peroxido } \\
(\mathrm{meq} / \mathrm{kg})\end{array}$ \\
\hline 0 & 0 & 1,98 \\
\hline 1 & 15 & 3,95 \\
\hline 2 & 30 & 5,02 \\
\hline 3 & 45 & 9,92 \\
\hline 4 & 60 & 13,83 \\
\hline 5 & 75 & 14,07 \\
\hline 6 & 90 & 15,82 \\
\hline 7 & 105 & 19,78 \\
\hline
\end{tabular}

Fonte: Os Autores, 2016.

De acordo com a Tabela acima observa-se que inicialmente o índice de peróxido é de $1,98 \mathrm{meq} / \mathrm{kg}$, dentro das recomendações estabelecidas pela Anvisa [11], cujo valor máximo deve ser de $20 \mathrm{meq} / \mathrm{kg}$. O aumento deste índice já era esperado uma vez que a fritura desencadeia um processo oxidativo com formação de peróxidos e hidroperóxidos [12]. Entretanto como o tempo de fritura foi curto em cada etapa, não observamos resultados acima do permitido pela legislação. Em outras análises realizadas no laboratório, onde o azeite de oliva foi submetido a períodos mais longos de aquecimento (entre 24-72 horas), mediu-se valores do índice de peróxido que chegaram a $123 \mathrm{meq} / \mathrm{kg}$, quando o azeite foi exposto a $100^{\circ} \mathrm{C}$ por 72 horas contínuas, permitindo supor que os índices de peróxido do azeite usado na fritura não alcançaram valores mais altos devido ao pouco tempo de aquecimento. 
Para os índices de acidez e peróxido considerou-se as amostras dos sete dias de fritura, entretanto para os espectros de absorção considerou-se apenas os cinco primeiros dias uma vez que os dois últimos dias não apresentaram mais a presença dos componentes moleculares identificados neste trabalho ( $\beta$-caroteno e clorofila A), supondo-se que tais degradaram em decorrência das sucessivas frituras.

\section{III.3. ESPECTROS DE ABSORÇÃO ÓPTICA}

Os espectros de absorção mostram apenas as bandas espectrais correspondentes às absorções de $\beta$-caroteno e clorofila A que, segundo [4] são os componentes relevantes para análise da degradação do azeite de oliva no que tange analises por espectroscopia óptica. As Figuras 1 e 2 mostram os espectros das diferentes amostras de azeite de oliva entre $375 \mathrm{~nm}$ e $550 \mathrm{~nm}$ e entre $630 \mathrm{~nm}$ e $700 \mathrm{~nm}$, respectivamente. Em ambas é observada a mudança sofrida pelos carotenos e clorofila ao longo das cinco etapas de fritura.

Figura 1: Espectros de absorção do azeite de oliva na região espectral de 375 a $550 \mathrm{~nm}$. 0: sem fritura; $1: 180^{\circ} / 15 \mathrm{~min} ; 2: 180^{\circ} \mathrm{C} / 30 \mathrm{~min}$; 3: $180^{\circ} \mathrm{C} / 45 \mathrm{~min}$; $4: 180^{\circ} \mathrm{C} / 60 \mathrm{~min}, 5: 180^{\circ} \mathrm{C} / 75 \mathrm{~min}$.

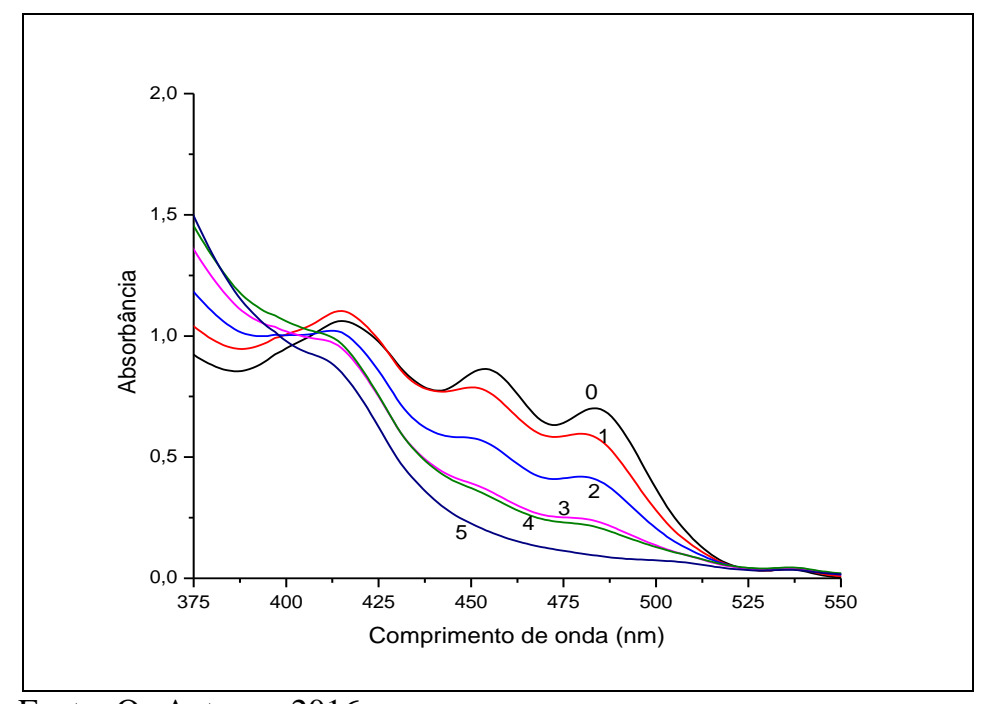

Fonte: Os Autores, 2016.

Na figura 1 observa-se uma banda lateral com uma estrutura tripla entre 400-500nm, onde são identificadas as assinaturas de clorofila A com máximo em torno de $415 \mathrm{~nm}$ e carotenoides, com prevalência de $\beta$-caroteno, em torno de 453 e
$483 \mathrm{~nm}$, respectivamente [4, 9] (espectros 0 e 2) que começam a deslocar para o azul e diminuir de intensidade à medida que o azeite vai sendo aquecido em frituras por dias seguidos (espectros 3-5). Esta é uma primeira evidência espectroscópica de modificação sofrida pelo azeite de oliva ao longo do tempo de uso em fritura.

Figura 2: Espectros de absorção do azeite de oliva nas regiões de 630-700 nm, com presença predominante de clorofila A em 670 nm. 1: $180^{\circ} / 15 \mathrm{~min} ; 2: 180^{\circ} \mathrm{C} / 30 \mathrm{~min} ; 3: 180^{\circ} \mathrm{C} / 45 \mathrm{~min} ; 4: 180^{\circ} \mathrm{C} / 60 \mathrm{~min}, 5: 180^{\circ} \mathrm{C} / 75 \mathrm{~min}$.



Fonte: Os Autores, 2016. 
Na figura 2, observa-se uma banda espectral com máximo em torno de 670nm atribuída à presença de clorofila A [4]. Vê-se que, à medida que o azeite usado em fritura ao longo do tempo, essa banda diminui de intensidade, sugerindo uma diminuição na quantidade líquida de clorofila no azeite. Este seria o segundo indicativo da mudança na composição molecular do azeite quando submetido a frituras em intervalos de tempo sucessivos.

Ao compararmos as medidas dos índices de acidez e peróxido (Tabelas 2 e 3), com os espectros de absorção das Figuras 1 e 2, observa-se que quando a absorção diminui os valores dos índices aumentam. Este padrão sugere que à medida que o $\beta$ caroteno, que atua como antioxidante, vai desaparecendo de maneira mais acelerada quando exposto a temperaturas acima de $40^{\circ} \mathrm{C}$, vai perdendo sua relevância como inibidor de oxidação. Ao mesmo tempo, a diminuição da quantidade de clorofila no azeite, por ação simultânea da temperatura e da luz ambiente, contribui para aceleração no processo oxidativo, por causa da formação do oxigênio singleto e de hidroperóxidos. A perda do $\beta$-caroteno e clorofila A precursores do processo oxidativo, vai desencadeando um tipo de auto-oxidação com formação de radicais livres [4, 5, 6, $7,11]$

\section{IV.CONCLUSÕES}

Verificou-se que o azeite de oliva submetido a frituras por longo tempo tem suas características químicas modificadas pela redução da absorção ótica de seus principais pigmentos - clorofilas e $\beta$-carotenos. As medidas dos índices de acidez e peróxido indicam que estas reduções estão relacionadas com o a formação de oxigênio singleto, hidroperóxidos e radicais livres. Os espectros de absorção UV-VIS das amostras de azeite de oliva aquecidos em frituras, mostraram a diminuição progressiva das quantidades de clorofilas e $\beta$-carotenos, que estão correlacionadas diretamente com os processos oxidativos que levam à degradação das propriedades alimentícias do azeite, tais como sabor, cor, cheiro e outros fatores relacionados à saúde humana. Por se tratar de uma técnica rápida, não invasiva e eficiente, sugere-se o uso das bandas espectrais do $\beta$-caroteno e clorofila A como indicadores de decomposição.

\section{REFERÊNCIAS}

[1] LOTTENBERG, A. M. P. Importância da gordura alimentar na prevenção e no controle de distúrbios metabólicos e da doença cardiovascular, Arq Bras Endocrinol. Metab. 53, 595607, 2009

[2] RAO, C. V.; NEWMARK, H. L., REDDY, B. S. Chemopreventive Effect of Squalene on Colon Cancer. Carcinogenesis 19, 287-290, 1998.

[3] BLEKAS, G.; TSIMIDOU, M.; BOSKOU, D. Olive Oil Chemistry and Technology, 2nd Edition, Dimitrios Boskou ed. AOCS Publishing, 41-71, 2006.

[4] DOMENICI, V.; ANCORA, D.; Cifelli, M.; SERANI, A.; VERACINI, C.A.; ZANDOMENEGHI, M., Extraction of pigment information from Near-UV Vis Absorption Spectra of
Extra Virgin Olive Oils. Journal of Agricultural and Food Chemistry 62, 9317-9325, 2004.

[5] RAHMANI, M.; CSALLANY, S. Role of Minor Constituents in the Photooxidation of Virgin Olive Oil. Department of Food Science and Nutrition, University of Minnesota, St. Paul, Minnesota. JAOCS, Vol. 75, no. 7, 1998

[6] HENRY, L. K.; CATIGNANI, G. L.; SCHWARTZ, S. J. Oxidative Degradation Kinetics of Lycopene, Lutein, and 9-cis and All-trans $\boldsymbol{\beta}$-Carotene. Department of Food Science, North Carolina State University, Raleigh, North Carolina. JAOCS, Vol. 75 , no. $7,1998$.

[7] DAMY, P. C.; JORGE, N., Determinação físico-químicas de óleo de soja e da gordura vegetal hidrogenada durante o processo de fritura descontinuo. Brazilian Journal of Food Technology. 6, 251-257, 2003.

[8] MELLO, L.D.; PINHEIRO, M.F., Aspectos Físico-Químicos de azeite de oliva e de folha de oliveira provenientes de cultivares do RS, Brasil. Revista Alim. Nutr. Araraquara/ SP 23 , 537-548, 2012.

[9] ALBUQUERQUE, M. L. S.; GUEDES, I.; Alcantara, P.; MOREIRA, S. G. C.; BARBOSA, N.M.; CORREA, Daniel S.; ZILIO, Sergio C. Characterization of Buriti (Mauritia flexuosa L.) Oil by Absorption and Emission Spectroscopies. J. Braz. Chem. Society 16,1114-1117, 2005.

[10] Instituto Adolfo Lutz. Métodos físico-químicos para análises de alimentos. Coordenadores: Odair Zenebon; Neus Sadocco Pascuet e Paulo Tiglea. IV Edição. São Paulo, 2008.

[11] ANVISA. Resolução $\mathbf{n}^{0}$ 482, de 23 de setembro de 1999. Regulamento técnico para fixação de identidade e qualidade de óleos e gorduras vegetais. Diário Oficial da República Federativa do Brasil, Brasília, 82-87, 1999

[12] SARGES, M.S.R. Análise da estabilidade oxidativa da oleína de palma com a adição dos antioxidantes naturais (tocoferóis e carotenoides) presentes no óleo de buriti, durante o processo de fritura. Universidade Federal do Pará, 2012. Publicado no $53^{\circ}$ Congresso Brasileiro de Química, 2013.

[13] JORGE, N.; SOARES, B.B.P.; LUNARD, V.M.; MALACRIDA, C.R., Alterações Físico-Químicas dos óleos de Girassol, milho e soja em fritura. Química Nova 28, 947-951, 2005 .

[14] TOLENTINO, M. C.; Kanumfre, F.; Bersot, L. S.; Nagata, N.; Carneiro, P. I. B.; Rosso, N. D., Avaliação da estabilidade fotooxidativa dos óleos de canola e de milho em presença de antioxidantes sintéticos. Ciência Rural 44, 4, 728-733, 2014. 